Article

\title{
A Note on Ricci Solitons
}

\author{
Sharief Deshmukh *(i) and Hana Alsodais (D) \\ Department of Mathematics, College of science, King Saud University, P.O. Box-2455, \\ Riyadh 11451, Saudi Arabia; halsodais@ksu.edu.sa \\ * Correspondence: shariefd@ksu.edu.sa
}

Received: 25 January 2020; Accepted: 14 February 2020; Published: 17 February 2020

check for updates

\begin{abstract}
In this paper, we characterize trivial Ricci solitons. We observe the important role of the energy function $f$ of a Ricci soliton (half the squared length of the potential vector field) in the charectrization of trivial Ricci solitons. We find three characterizations of connected trivial Ricci solitons by imposing different restrictions on the energy function. We also use Hessian of the potential function to characterize compact trivial Ricci solitons. Finally, we show that a solution of a Poisson equation is the energy function $f$ of a compact Ricci soliton if and only if the Ricci soliton is trivial.
\end{abstract}

Keywords: ricci soliton; energy function; Trivial Ricci soliton; poisson equation

\section{Introduction}

Ricci solitons are self-similar solutions of the Ricci flow (cf. [1]), and occupy a position of immense importance in differential geometry. Ricci solitons are important as they have been used to settle a century old conjecture namely the Poincare conjecture, (for other important roles of Ricci soliton see the Conclusion-section). An $n$-dimensional Riemannian manifold $(M, g)$, may be compact or noncompact, is called a Ricci soliton, if there is a smooth vector field $\xi$ satisfying (cf. Reference [1]),

$$
\operatorname{Ric}+\frac{1}{2} £_{\xi} g=\lambda g
$$

where Ric is the Ricci tensor, $£_{\xi} g$ is the Lie derivative of the metric $g$ with respect to $\xi$ and $\lambda$ is a constant. Note that $\lambda$ can be allowed to be a function and in this case the Ricci soliton is called almost Ricci soliton. We denote a Ricci soliton by $(M, g, \xi, \lambda)$ and call $\xi$ the potential vector field of the Ricci soliton. A Ricci soliton $(M, g, \xi, \lambda)$ is said to be expanding, stable or shrinking depending on $\lambda<0$, $\lambda=0$ or $\lambda>0$ respectively. If the potential field $\xi$ is a gradient of a smooth function $h$ (that is, $\xi=\nabla h$ ), then $(M, g, \nabla h, \lambda)$ is called a gradient Ricci soliton and in this case Equation (1) takes the form

$$
\text { Ric }+H_{h}=\lambda g
$$

where $H_{h}$ is the Hessian of the function $h$. A compact Ricci soliton is always a gradient Ricci soliton (cf. Reference [1]). A Ricci soliton $(M, g, \xi, \lambda)$ is said to be trivial if $£_{\xi} g=0$, and in this case the metric $g$ is an Einstein metric with soliton constant becoming an Einstein constant. Geometry of Ricci solitons is highly pursued subject not only because of its elegant geometry, but also because of its applications in different disciplines. In Reference [2-4], Myers-type theorems have been proved for Ricci solitons. Also, in Reference [5], it has been proved that a complete shrinking Ricci soliton $(M, g, \xi, \lambda)$ has finite fundamental group. In Reference [6,7], volume comparison theorems of Bishop-type are derived for noncompact shrinking Ricci solitons.

The importance of obtaining conditions for a Ricci soliton to be trivial comes from the fact that Ricci solitons are a generalization of Einstein manifolds. Recently, the authors of Reference [8,9] have found characterizations of trivial Ricci solitons. In this paper, we find necessary and sufficient 
conditions for Ricci solitons to be trivial in both compact and noncompact cases. Given a Ricci soliton $(M, g, \xi, \lambda)$, we have a smooth function $f=\frac{1}{2}\|\xi\|^{2}$, which we call the energy function of the Ricci soliton. We observe that the energy function of the Ricci soliton, plays an important role in obtaining characterizations of a trivial Ricci soliton. In our first result, we show that on a connected Ricci soliton $(M, g, \xi, \lambda)$ the Laplacian $\Delta f$ of the energy function, bounded above by some geometric quantity, gives a characterization of a connected trivial Ricci soliton (cf. Theorem 1). We also show that for a certain Ricci soliton $(M, g, \xi, \lambda)$, the scalar curvature is a constant along integral curves of potential field $\xi$ and the energy function is superharmonic, gives another characterization of a connected Ricci soliton (cf. Theorem 2). Also, we observe that, invariance of the Ricci operator of the Ricci soliton under the local flow of the potential vector field is useful in characterizing a connected trivial Ricci soliton (cf. Theorems 3 and 4). Then, regarding compact Ricci solitons $(M, g, \nabla h, \lambda)$, we use the Hessian of the potential function $H_{h}$ to find a characterization of a compact Ricci soliton (cf. Theorem 1). Finally, we show that on a compact shrinking Ricci soliton $(M, g, \nabla h, \lambda)$ with energy function $f$, the function $\frac{1}{\lambda} f$ satisfies Poisson equation if and only if the Ricci soliton is trivial (cf. Theorem 2).

\section{Preliminaries}

Let $(M, g, \xi, \lambda)$ be an $n$-dimensional Ricci soliton. We denote by $\eta$ the smooth 1 -form dual to the potential field $\xi$. Then, we define a skew symmetric tensor field $\varphi$ on the Ricci soliton $(M, g, \xi, \lambda)$ by

$$
\frac{1}{2} d \eta(X, Y)=g(\varphi X, Y), \quad X, Y \in \mathfrak{X}(M),
$$

where $\mathfrak{X}(M)$ is the Lie algebra of smooth vector fields on $M$. We call this tensor field $\varphi$ the associated tensor field of the Ricci soliton $(M, g, \xi, \lambda)$. Using above equation and Equation (1) in Koszul's formula (cf. Reference [10]), the covariant derivative of the potential field $\xi$ is given by

$$
\nabla_{X} \xi=\lambda X-Q X+\varphi X, \quad X \in \mathfrak{X}(M),
$$

where $Q$ is the Ricci operator of the Ricci soliton $(M, g, \xi, \lambda)$, which is a symmetric operator that is related to Ricci tensor Ric by

$$
\operatorname{Ric}(X, Y)=g(Q X, Y), \quad X, Y \in \mathfrak{X}(M) .
$$

The scalar curvature $S$ of the Ricci soliton $(M, g, \xi, \lambda)$ is given by $S=\operatorname{Tr} Q$, and its gradient $\nabla S$ satisfies

$$
\sum_{i=1}^{n}(\nabla Q)\left(e_{i}, e_{i}\right)=\frac{1}{2} \nabla S,
$$

where $\left\{e_{1}, . ., e_{n}\right\}$ is a local orthonormal frame and $(\nabla Q)(X, Y)=\nabla_{X} Q Y-Q\left(\nabla_{X} Y\right)$.

Using the definition of the curvature tensor field

$$
R(X, Y) Z=\nabla_{X} \nabla_{Y} Z-\nabla_{Y} \nabla_{X} Z-\nabla_{[X, Y]} Z, \quad X, Y, Z \in \mathfrak{X}(M) .
$$

and Equation (3), we get the following expression for curvature tensor of $(M, g, \xi, \lambda)$ :

$$
R(X, Y) \xi=(\nabla Q)(Y, X)-(\nabla Q)(X, Y)+(\nabla \varphi)(X, Y)-(\nabla \varphi)(Y, X) .
$$

Using symmetry of $Q$, skew-symmetry of the associated tensor field $\varphi$, and Equations (4) and (5), we obtain

which leads to

$$
\operatorname{Ric}(Y, \xi)=Y(S)-\frac{1}{2} Y(S)-g\left(Y, \sum(\nabla \varphi)\left(e_{i}, e_{i}\right)\right),
$$

$$
Q(\xi)=\frac{1}{2} \nabla S-\sum(\nabla \varphi)\left(e_{i}, e_{i}\right)
$$


Define a smooth function $f$ on $(M, g, \xi, \lambda)$ by $f=\frac{1}{2}\|\xi\|^{2}$ and call it energy function of the Ricci soliton. Using Equation (3), it follows that the gradient of the energy function $f$ is given by

$$
\nabla f=\lambda \xi-Q \xi-\varphi \xi
$$

The Hessian operator $A_{f}$ of the energy function $f$ is given by

$$
A_{f} X=\nabla_{X} \nabla f, \quad X \in \mathfrak{X}(M)
$$

and the Laplacian $\Delta f=\operatorname{div}(\nabla f)$ satisfies $\Delta f=\operatorname{Tr} A_{f}$. Moreover, the Hessian of the energy function $f$ is given by

$$
H_{f}(X, Y)=g\left(A_{f} X, Y\right), \quad X \in \mathfrak{X}(M) .
$$

Using Equations (3), (4) and (6), we have

$$
\operatorname{div} \xi=n \lambda-S, \quad \operatorname{div} Q \xi=\lambda S-\|Q\|^{2}+\frac{1}{2} \xi(S)
$$

and

$$
\operatorname{div} \varphi \xi=-\|\varphi\|^{2}+\operatorname{Ric}(\xi, \xi)-\frac{1}{2} \xi(S)
$$

Note that we have used the symmetry of $Q$, skew symmetry of $\varphi$ and that

$$
\|\varphi\|^{2}=\sum_{i=1}^{n} g\left(\varphi e_{i}, \varphi e_{i}\right)
$$

for a local orthonormal frame $\left\{e_{1}, . ., e_{n}\right\}$ on $n$-dimensional Ricci soliton $(M, g, \xi, \lambda)$.

Lemma 1. The Hessian operator $A_{f}$ of the energy function $f$ on the Ricci soliton $(M, g, \xi, \lambda)$ is given by

$$
A_{f} X=(Q-\lambda I)^{2} X-\varphi^{2} X+\varphi Q X-Q \varphi X-(\nabla Q)(X, \xi)-(\nabla \varphi)(X, \xi), X \in \mathfrak{X}(M) .
$$

Proof. Note that, using Equation (3), we have

$$
\begin{aligned}
\nabla_{X} Q \xi & =(\nabla Q)(X, \xi)+Q(\lambda X-Q X+\varphi X) \\
& =(\nabla Q)(X, \xi)+\lambda Q X-Q^{2} X+Q \varphi X
\end{aligned}
$$

Similarly, we have

$$
\nabla_{X} \varphi \xi=(\nabla \varphi)(X, \xi)+\lambda \varphi X-\varphi Q X+\varphi^{2} X
$$

Inserting these values in the covariant derivative of Equation (7) and using Equation (3), we get the expression for $A_{f} X$, required in the Lemma.

\section{Characterizations of Connected Trivial Ricci Solitons}

Since compact Ricci solitons are gradient Ricci solitons and therefore, the potential vector field $\xi$ is closed, which forces the associated tensor field $\varphi=0$. Thus, many equations dealing with the geometry of compact Ricci soliton get simplified apart from this, many other tools such as Stokes theorem and critical point theory is also available on compact Ricci solitons. The study of non-compact Ricci solitons lacks these tools. In this section, we obtain several necessary and sufficient conditions for a connected Ricci soliton to be a trivial Ricci soliton. Our first result is the following: 
Theorem 1. An n-dimensional connected Ricci soliton $(M, g, \xi, \lambda)$ with energy function $f$ and associated tensor field $\varphi$, is trivial if and only if

$$
\Delta f \leq\|\varphi\|^{2}-\operatorname{Ric}(\xi, \xi)
$$

Proof. Suppose $(M, g, \xi, \lambda)$ is connected, that satisfies the inequality

$$
\Delta f \leq\|\varphi\|^{2}-\operatorname{Ric}(\xi, \xi) .
$$

Now, for a local orthonormal frame $\left\{e_{1}, . ., e_{n}\right\}$ on $(M, g, \xi, \lambda)$, by Lemma 1 , we get

$$
\begin{aligned}
\Delta f= & n \lambda^{2}-2 \lambda S+\|Q\|^{2}+\|\varphi\|^{2}-g\left(\xi, \sum_{i=1}^{n}(\nabla Q)\left(e_{i}, e_{i}\right)\right) \\
& +g\left(\xi, \sum_{i=1}^{n}(\nabla \varphi)\left(e_{i}, e_{i}\right)\right),
\end{aligned}
$$

where, we used $\operatorname{Tr} Q \varphi=\operatorname{Tr} \varphi Q=0$. Using Equations (4) and (6) in above equation, we conclude

$$
\Delta f=\left(\|Q\|^{2}-\frac{1}{n} S^{2}\right)+\frac{1}{n}(n \lambda-S)^{2}+\|\varphi\|^{2}-\operatorname{Ric}(\xi, \xi) .
$$

Now, using inequality (10) and Schwartz inequality $\|Q\|^{2} \geq \frac{1}{n} S^{2}$, in Equation (11), we conclude that

$$
\|Q\|^{2}-\frac{1}{n} S^{2}=0 \text { and } S=n \lambda .
$$

However, the equality in Schwartz inequality holds if and only if $Q=\frac{S}{n} I$, and using $S=n \lambda$, gives $Q=\lambda I$. Hence, $R i c=\lambda g$, that is $(M, g, \xi, \lambda)$ is trivial.

Conversely, if $(M, g, \xi, \lambda)$ is trivial, then $Q \xi=\lambda \xi$ and Equation (7), gives $\nabla f=-\varphi \xi$. Moreover, as $S$ is a constant, Equation (9), gives

$$
\operatorname{div} \varphi \xi=-\|\varphi\|^{2}+\operatorname{Ric}(\xi, \xi)
$$

and consequently,

$$
\Delta f=\|\varphi\|^{2}-\operatorname{Ric}(\xi, \xi)
$$

holds.

If the Laplacian of a smooth function $h$ is identically zero, then $h$ is said to be harmonic. It is subharmonic if it is non-negative, whereas it is superharmonic if the Laplacian is non-positive. In the following, we give a characterization of a connected trivial soltion using the super harmonicity of the energy function.

Theorem 2. Let $(M, g, \xi, \lambda)$ be an n-dimensional connected Ricci soliton with the vector field $\varphi \xi$ divergence free. Then $(M, g, \xi, \lambda)$ is trivial if and only if, the scalar curvature $S$ is a constant along the integral curves of potential vector field $\xi$ and the energy function $f$ is superharmonic.

Proof. Suppose $(M, g, \xi, \lambda)$ is connected and satisfies

$$
\operatorname{div} \varphi \xi=0, \xi(S)=0 \text { and } \Delta f \leq 0 .
$$


Then Equation (9), implies $\operatorname{Ric}(\xi, \xi)=\|\varphi\|^{2}$ and consequently, Equation (11) takes the form

$$
\Delta f=\left(\|Q\|^{2}-\frac{1}{n} S^{2}\right)+\frac{1}{n}(n \lambda-S)^{2} .
$$

Since, $f$ is superharmonic, above equation gives $Q=\lambda I$, that is, $(M, g, \xi, \lambda)$ is trivial.

Conversely, if $(M, g, \xi, \lambda)$ is trivial with $\operatorname{div} \varphi \xi=0$, then $S$ is a constant and Equation (7) gives $\nabla f=-\varphi \xi$. Consequently, we have $\Delta f=0$, that is, $f$ is harmonic and therefore superharmonic.

Recall that a vector field $X$ on a Riemannian manifold $(M, g)$ with local flow $\left\{\psi_{t}\right\}$, a $(1,1)$ tensor field $\Omega$ on $(M, g)$ is said to be invariant under the flow of $X$ if $d \psi_{t} \circ \Omega=\Omega \circ d \psi_{t}$ holds. Also, note that if $(M, g, \xi, \lambda)$ is trivial, then the potential vector field $\xi$ is a Killing vector field and consequently, the local flow of $\xi$ consists of local isometries of $(M, g, \xi, \lambda)$ and therefore the Ricci operator $Q$ is invariant under the local flow of $\xi$. A natural question arises: Is a Ricci soliton $(M, g, \xi, \lambda)$ with Ricci operator $Q$ invariant under the local flow of the potential vector field $\xi$, necessarily trivial? Answer to this question is in negative, for the Ricci soliton $\left(R^{n},\langle\rangle,, \xi, \lambda\right), \xi=\psi, \lambda=1$ is a nontrivial Ricci soliton with Ricci operator invariant under the flow of $\xi$, where $\psi$ is the position vector field on $R^{n}$. Using this notion, we prove the following.

Theorem 3. Let $(M, g, \xi, \lambda)$ be an n-dimensional connected Ricci soliton. Then $(M, g, \xi, \lambda)$ is trivial if and only if, the Ricci operator $Q$ is invariant under the local flow of the potential vector field $\xi$ and

$$
Q \xi=\lambda \xi
$$

Proof. Suppose $(M, g, \xi, \lambda)$ be an $n$-dimensional connected Ricci soliton with the Ricci operator $Q$ invariant under the local flow of the potential vector field $\xi$. Then we have

$$
£_{\xi} Q=0,
$$

which in view of (3), gives

$$
(\nabla Q)(\xi, X)=\varphi Q X-Q \varphi X, \quad X \in \mathfrak{X}(M) .
$$

Taking $X=e_{i}$ in above equation and the inner product with $e_{i}$, and summing over a local orthonormal frame $\left\{e_{1}, \ldots, e_{n}\right\}$, we conclude, $\xi(S)=0$. Now, taking divergence on both sides of the equation $Q \xi=\lambda \xi$ and using Equation (8), we conclude

$$
\lambda(n \lambda-S)=\lambda S-\|Q\|^{2},
$$

that is,

$$
\left(\|Q\|^{2}-\frac{1}{n} S^{2}\right)+\frac{1}{n}(n \lambda-S)^{2}=0 .
$$

Hence, we get Ric $=\lambda g$, that is, $(M, g, \xi, \lambda)$ is trivial.

Conversely, if $(M, g, \xi, \lambda)$ is trivial, then Ric $=\lambda g$ and that $\xi$ is Killing. Thus, the local flow of $\xi$ consists of local isometries of $(M, g, \xi, \lambda)$ and therefore $Q$ is invariant under the local flow of $\xi$ and $Q \xi=\lambda \xi$ holds.

Theorem 4. Let $(M, g, \xi, \lambda)$ be an n-dimensional connected Ricci soliton. Then $(M, g, \xi, \lambda)$ is trivial if and only if, the Ricci operator $Q$ is invariant under the local flow of the potential vector field $\xi$ and the energy function $f$ is a constant along the integral curves of $\xi$. 
Proof. Suppose $(M, g, \xi, \lambda)$ is connected such that $Q$ is invariant under the local flow of $\xi$ and that $\xi(f)=0$ holds. Then using Lemma 1 and Equation (13), we conclude

$$
A_{f} \xi=(Q-\lambda I)^{2} \xi-\varphi^{2} \xi-(\nabla \varphi)(\xi, \xi) .
$$

Taking the inner product with $\xi$ in above equation and noting that $Q-\lambda I$ is symmetric and $\varphi$ is skew-symmetric, we get

$$
H_{f}(\xi, \xi)=\|Q \xi-\lambda \xi\|^{2}+\|\varphi \xi\|^{2} .
$$

Note that, using Equation (3), we have $\nabla_{\xi} \xi=\lambda \xi-Q \xi+\varphi \xi$. Thus, using Equation (7), we compute

$$
\nabla_{\xi} \xi(f)=g(\lambda \xi-Q \xi+\varphi \xi, \lambda \xi-Q \xi-\varphi \xi),
$$

that is,

$$
\nabla_{\xi} \xi(f)=\lambda^{2}\|\xi\|^{2}-2 \lambda g(Q \xi, \xi)+\|Q \xi\|^{2}-\|\varphi \xi\|^{2}
$$

which yields

$$
\nabla_{\xi} \xi(f)=\|Q \xi-\lambda \xi\|^{2}-\|\varphi \xi\|^{2}
$$

Since, $\xi(f)=0$, we have $H_{f}(\xi, \xi)=-\nabla_{\xi} \xi(f)$, and consequently, using Equations (14) and (15), we get

$$
\|Q \xi-\lambda \xi\|^{2}=0,
$$

proving that $Q \xi=\lambda \xi$. Hence, Theorem 3 implies that $(M, g, \xi, \lambda)$ is trivial.

Conversely, if $(M, g, \xi, \lambda)$ is trivial, then as $\xi$ is Killing, we get that $Q$ is invariant under the local flow of $\xi$. Moreover, using $Q \xi=\lambda \xi$ in Equation (7), we get $\nabla f=-\varphi \xi$, which proves that the energy function $f$ is a constant along the integral curves of $\xi$.

We end this section by giving two examples where we analyze the conditions in the statements of the results above. The first of a trivial Ricci soliton whereas the second of a nontrivial soliton.

Example 1. (i) Consider the Euclidean space $\left(R^{2 n},\langle\rangle,\right)$ and the vector field $\xi=J \psi$, where $J$ is the complex structure on $R^{2 n}$ and $\psi$ is the position vector field. Then, we see that $\left(R^{2 n},\langle\rangle,, \xi, \lambda\right)$ is a trivial Ricci soliton with $\lambda=0$, the associated tensor field $\varphi=J$ and the energy function $f=\frac{1}{2}\|\psi\|^{2}$. Moreover, we find that $\|\varphi\|^{2}=2 n, \nabla f=\psi$ and that $\Delta f=2 n$. Hence,

$$
\Delta f=\|\varphi\|^{2}-\operatorname{Ric}(\xi, \xi),
$$

holds and $f$ is a subharmonic function. Also, we have $\xi(f)=0$ and $\operatorname{div} \varphi \xi=-2 n$ holds and as $Q=0$, the Ricci operator is invariant under the flow of $\xi$.

(ii) Consider the Euclidean space $\left(R^{n},\langle\rangle,\right)$ and the vector field

$$
\xi=\psi+x^{2} \frac{\partial}{\partial x^{1}}-x^{1} \frac{\partial}{\partial x^{2}} .
$$

Then, denoting by $\nabla$, the Euclidean connection on $\left(R^{n},\langle\rangle,\right)$, we find that

$$
\nabla_{X} \xi=X+\varphi X, \quad X \in \mathfrak{X}\left(R^{n}\right),
$$

where

$$
\varphi X=X\left(x^{2}\right) \frac{\partial}{\partial x^{1}}-X\left(x^{1}\right) \frac{\partial}{\partial x^{2}}
$$


which satisfies $\langle\varphi X, Y\rangle=-\langle X, \varphi Y\rangle$, that is, $\varphi$ is a skew-symmetric operator on $\left(R^{n},\langle\rangle,\right)$. It follows that $\left(R^{n},\langle\rangle,, \xi, \lambda\right), \lambda=1$ is a nontrivial Ricci soliton with potential vector field $\xi$, the associated tensor field $\varphi$ and the energy function $f=\frac{1}{2}\left(\|\psi\|^{2}+\left(x^{1}\right)^{2}+\left(x^{1}\right)^{2}\right)$. Moreover, we have

$$
\nabla f=\psi+x^{1} \frac{\partial}{\partial x^{1}}+x^{2} \frac{\partial}{\partial x^{2}}
$$

which gives $\xi(f)=2 f \neq 0$ and that $\Delta f=n+2$ holds. Also, we have $\|\varphi\|^{2}=2$, and consequently $\Delta f>\|\varphi\|^{2}-\operatorname{Ric}(\xi, \xi)=2$, that is $f$ is subharmonic. Also, we have div $\varphi \xi=0$ and as $Q=0$ it is invariant under the flow of the potential vector field $\xi$.

\section{Characterizations of Compact Trivial Ricci Solitons}

In this section, we consider a compact Ricci soliton $(M, g, \xi, \lambda)$ and find characterizations of a trivial Ricci soliton. As a compact Ricci soliton is a gradient Ricci soliton (cf. Reference [1]). Thus, if $(M, g, \xi, \lambda)$ is a gradient Ricci soliton, then $\xi=\nabla h$ for a smooth function $h$ and the Equation (2) holds, we call $h$ the potential function of the Ricci soliton. Moreover, for $(M, g, \nabla h, \lambda)$, as $\xi=\nabla h$ is closed, $\varphi=0$ and Equation (6) takes the form

$$
Q(\nabla h)=\frac{1}{2} \nabla S .
$$

Using above equation and Equation (2), we get (cf. Reference [1])

$$
2 \lambda h=\|\nabla h\|^{2}+S .
$$

Theorem 1. Let $(M, g, \nabla h, \lambda)$ be an $n$-dimensional compact shrinking Ricci soliton of positive Ricci curvature and the first nonzero eigenvalue $\lambda_{1}$ of the Laplace operator. Then, $(M, g, \nabla h, \lambda)$ is trivial if and only if the Hessian $\mathrm{H}_{h}$ satisfies the inequality

$$
H_{h}(\nabla S, \nabla S) \geq \lambda\left(2 \lambda-\lambda_{1}\right)(n \lambda-S)^{2} .
$$

Proof. Suppose $(M, g, \nabla h, \lambda)$ is an $n$-dimensional compact Ricci soliton of positive Ricci curvature with Hessian $H_{h}$ satisfying the inequality

$$
H_{h}(\nabla S, \nabla S) \geq \lambda\left(2 \lambda-\lambda_{1}\right)(n \lambda-S)^{2},
$$

where $\lambda_{1}$ is the first nonzero eigenvalue of the Laplace operator acting on smooth functions on $M$. The Equation (3) takes the form

$$
A_{h} X=\lambda X-Q(X), \quad X \in \mathfrak{X}(M),
$$

which gives

$$
\left\|A_{h}\right\|^{2}=n \lambda^{2}-2 \lambda S+\|Q\|^{2}, \quad \Delta h=n \lambda-S .
$$

Now, using Bochner's formula

$$
\int_{M}\left(\operatorname{Ric}(\nabla h, \nabla h)+\left\|A_{h}\right\|^{2}-(\Delta h)^{2}\right)=0,
$$

we get

$$
\int_{M}\left(R i c(\nabla h, \nabla h)+n \lambda^{2}-2 \lambda S+\|Q\|^{2}-(n \lambda-S)^{2}\right)=0,
$$


that is,

$$
\int_{M}\left(\operatorname{Ric}(\nabla h, \nabla h)+\left(\|Q\|^{2}-\frac{1}{n} S^{2}\right)-\frac{n-1}{n}(n \lambda-S)^{2}\right)=0 .
$$

For a gradient Ricci soliton, using Lemma 1, we have

$$
\Delta f=\left(\|Q\|^{2}-\frac{1}{n} S^{2}\right)+\frac{1}{n}(n \lambda-S)^{2}-R i c(\nabla h, \nabla h),
$$

which on integration, gives

$$
\int_{M}\left(\operatorname{Ric}(\nabla h, \nabla h)-\left(\|Q\|^{2}-\frac{1}{n} S^{2}\right)-\frac{1}{n}(n \lambda-S)^{2}\right)=0 .
$$

Adding Equations (21) and (22), we conclude

$$
\int_{M} \operatorname{Ric}(\nabla h, \nabla h)=\frac{1}{2} \int_{M}(n \lambda-S)^{2} .
$$

Now, Equation (16) gives

$$
\operatorname{Ric}(\nabla h, \nabla S)=\frac{1}{2}\|\nabla S\|^{2}
$$

and Equation (19) gives

$$
\operatorname{Ric}(\nabla S, \nabla S)=\lambda\|\nabla S\|^{2}-H_{h}(\nabla S, \nabla S)
$$

We have

$$
\operatorname{Ric}(2 \lambda \nabla h-\nabla S, 2 \lambda \nabla h-\nabla S)=4 \lambda^{2} \operatorname{Ric}(\nabla h, \nabla h)-4 \lambda \operatorname{Ric}(\nabla h, \nabla S)+\operatorname{Ric}(\nabla S, \nabla S),
$$

which on integrating and using Equations (23), (24) and (25) yields

$$
\begin{aligned}
& \int_{M} \operatorname{Ric}(2 \lambda \nabla h-\nabla S, 2 \lambda \nabla h-\nabla S) \\
= & \int_{M}\left(2 \lambda^{2}(n \lambda-S)^{2}-\lambda\|\nabla S\|^{2}-H_{h}(\nabla S, \nabla S)\right) .
\end{aligned}
$$

Using Equation (20), we have $\int_{M}(n \lambda-S)=0$, with $\nabla(n \lambda-S)=-\nabla S$, and consequently, using minimum principle, we conclude

$$
\int_{M}\|\nabla S\|^{2} \geq \lambda_{1} \int_{M}(n \lambda-S)^{2}
$$

Thus, as $\lambda>0$ (for shrinking Ricci soliton), using above inequality in Equation (26), we get

$$
\int_{M} \operatorname{Ric}(2 \lambda \nabla h-\nabla S, 2 \lambda \nabla h-\nabla S) \leq \int_{M}\left(\lambda\left(2 \lambda-\lambda_{1}\right)(n \lambda-S)^{2}-H_{h}(\nabla S, \nabla S)\right) .
$$

Since, Ricci curvature is positive, using inequality (18) in above inequality, we get $2 \lambda \nabla h=\nabla S$, that is, $\nabla(2 \lambda h-S)=0$. Thus, $2 \lambda h-S=c$, where $c$ is a constant. Now, invoking Equation (17), we conclude $\|\nabla h\|^{2}=c$ and as $M$ is compact, at a critical point of $h$, we get $c=0$. Hence $\nabla h=0$, that is, $(M, g, \nabla h, \lambda)$ is trivial.

The converse is trivial. 
Given a compact $(M, g, \nabla h, \lambda)$, we have

$$
\int_{M}(n \lambda-S)=0
$$

and consequently, the Poisson equation $\Delta \sigma=(n \lambda-S)$ has a unique solution on $(M, g, \nabla h, \lambda)$ up to a constant (cf. Reference [11]). Now, we prove the following:

Theorem 2. Let $(M, g, \nabla h, \lambda)$ be an $n$-dimensional compact shrinking Ricci soliton with energy function $f$. Then,$(M, g, \nabla h, \lambda)$ is trivial if and only if, $\frac{1}{\lambda} f$ is a solution of the Poisson equation

$$
\Delta \sigma=(n \lambda-S)
$$

Proof. Suppose $(M, g, \nabla h, \lambda)$ is an $n$-dimensional compact shrinking Ricci soliton with energy function $f$, such that $\frac{1}{\lambda} f$ satisfies Poisson equation $\Delta \sigma=(n \lambda-S)$. Then using Equation (20), $\Delta h=n \lambda-S$ and Donaldson's result (cf. Reference [11]), we conclude $\frac{1}{\lambda} f=h+c$, where $c$ is a constant. This proves $\nabla f=\lambda \nabla h$. However, Equation (7) for a gradient soliton reads

$$
\nabla f=\lambda \nabla h-Q(\nabla h)
$$

which gives $Q(\nabla h)=0$. Then, Equation (16) confirms that $S$ is a constant. Thus, integrating equation $\Delta h=n \lambda-S$, we get $S=n \lambda$, which proves $\Delta h=0$, that is, $h$ is a constant. Hence, Equation (2) gives Ric $=\lambda g$, that is, $(M, g, \nabla h, \lambda)$ is trivial.

Conversely, if $(M, g, \nabla h, \lambda)$ is trivial, then Equation (2) proves that $h$ is a constant and we get $f=0$, which satisfies the Poisson equation $\Delta \sigma=0$.

Theorem 3. An n-dimensional compact Ricci soliton $(M, g, \nabla h, \lambda)$ is trivial if and only if, the Ricci operator $Q$ is invariant under the flow of the potential vector field $\nabla h$.

Proof. Suppose $Q$ is invariant under the flow of the potential vector field $\nabla h$.

$$
£_{\nabla h} Q=0,
$$

and using (19) gives $(\nabla Q)(\nabla h, X)=0, X \in \mathfrak{X}(M)$. Thus, we conclude that $g(\nabla h, \nabla S)=0$, and consequently, Equation (16) gives $\operatorname{Ric}(\nabla h, \nabla h)=0$. Now, using this information and Lemma 1, for a gradient Ricci soliton, leads to

$$
\Delta f=\left(\|Q\|^{2}-\frac{1}{n} S^{2}\right)+\frac{1}{n}(n \lambda-S)^{2} .
$$

Integrating above equation, we conclude $\operatorname{Ric}=\lambda g$, that is, $(M, g, \nabla h, \lambda)$ is trivial.

The converse is trivial.

Suppose on compact $(M, g, \nabla h, \lambda), Q$ satisfies $Q(\nabla h)=\lambda \nabla h$. Then, using Equation (16), we get $\nabla(2 \lambda h-S)=0$, that is, $2 \lambda h-S=c$ for a constant $c$. Thus, Equation (17) implies that $\|\nabla h\|^{2}=c$ on compact $M$, which gives $\nabla h=0$ and this proves that $(M, g, \nabla h, \lambda)$ is trivial. The converse is also true. Hence, we have the following:

Corollary 1. An n-dimensional compact Ricci soliton $(M, g, \nabla h, \lambda)$ is trivial if and only if, the Ricci operator $Q$ satisfies

$$
Q(\nabla h)=\lambda \nabla h
$$




\section{Conclusions}

A Ricci soliton is a self similar solution of the Ricci flow and its first importance calme from the fact that it was applied in settling the century old Poincare conjecture and later it became a central tool in applications to various areas of sciences and economics. Ricci solitons are useful in physics (cf. Reference [12]), in biology, chemistry (cf. Reference [13]) and economics (cf. Reference [14]). Moreover, Ricci flow and Ricci solitons have shown their presence in medical imaging for brain surfaces (cf. Reference [15]). In a Ricci soliton $(M, g, \xi, \lambda)$, if the constant $\lambda$ is allowed to be a smooth function, then $(M, g, \xi, \lambda)$ is called an almost Ricci soliton (cf. Reference [16]) and these days almost Ricci soliton is subject of interest. As the Ricci flow is a heat equation satisfied by the evolving metric using Ricci curvature, its natural extension is the Yamabe flow where the evolving metric satisfies the partial differential equation

$$
\frac{\partial g_{t}}{\partial t}=-S(t) g_{t}
$$

where $S(t)$ is the scalar curvature of the evolving metric $g_{t}$. Stable solutions of Yamabe flow are called Yamabe solitons (cf. Reference [6,17]) A stablesolution of the Yamabe soliton is denoted by $(M, g, \mathbf{u}, \lambda)$, where the potential filed $\mathbf{u}$ satisfies

$$
\frac{1}{2} £_{\mathbf{u}} g=(S-\lambda) g
$$

for a constant $\lambda$. Trivial Yamabe solitons are Riemannian manifolds of constant scalar curvature. If in a Yamabe soliton $(M, \mathbf{u}, \xi, \lambda)$, the constant $\lambda$ is allowed to become a smooth function, it is called almost Yamabe solitons. In geometry of Ricci solitons, almost Ricci solitons, Yamabe solitons as well as almost Yamabe solitons an important question is to find conditions under which they become trivial Ricci solitons, trivial Yamabe solitons respectively. Our results are important steps in this direction.

Author Contributions: Both authors have contributed equally in this paper and agree to the published version of the manuscript.

Funding: This research project was supported by a grant from the "Research Center of the Female Scientific and Medical Colleges", Deanship of Scientific Research, King Saud University.

Conflicts of Interest: The authors declare no conflict of interest.

\section{References}

1. Chow, B.; Lu, P.; Ni, L. Hamilton's Ricci flow: Graduate studies in Mathematics. In Hamilton's Ricci Flow: Graduate Studies in Mathematics; AMS Providence: Providence, RI, USA, 2006.

2. Derdzinski, A. A Myers-type theorem and compact Ricci solitons. Proc. Am. Math. Soc. 2006, 134, 3645-3648. [CrossRef]

3. Munteanu, O.; Wang, J. Geometry of shrinking Ricci solitons. Comp. Math. 2015, 151, 2273-2300. [CrossRef]

4. Munteanu, O.; Wang, J. Positively curved shrinking Ricci solitons are compact. J. Diff. Geom. 2017, 106, 499-505. [CrossRef]

5. Wylie, W. Complete shrinking Ricci solitons have finite fundamental group. Proc. Am. Math. Soc. 2008, 136, 1803-1806. [CrossRef]

6. Cao, H.-D. Geometry of Ricci solitons. Chin. Ann. Math. 2006, 27B, 121-142. [CrossRef]

7. Cao, H.-D.; Zhou, D. On complete gradient shrinking Ricci solitons. Diff. Geom. 20010, 85, $175-185$. [CrossRef]

8. Deshmukh, S. Jacobi-type vector fields on Ricci solitons. Bull. Math. Soc. Sci. Math. Roum. 2012, 1, 41-50.

9. Li, F.; Zhou, J. Rigidity characterization of compact Ricci solitons. J. Korean Math. Soc. 2019, 56. [CrossRef]

10. Lee, J.M. Riemannian Manifolds. In Riemannian Manifolds; Springer: Berlin/Heidelberg, Germany, 1997.

11. Donaldson, S. Geometric Analysis. In Lecture Notes for TCC Course "Geometric Analysis". Available online: http:/ / wwwf.imperial.ac.uk/ skdona/GEOMETRICANALYSIS.PDF (accessed on 16 February 2020).

12. Graf, W. Ricci flow gravity. PMC Phys. A 2007, 1, 3. [CrossRef]

13. Ivancevic, V.G.; Ivancevic, T.T. Ricci flow and nonlinear reaction-diffusion systems in biology, chemistry, and physics. Nonliear Dyn. 2011, 65, 35-54. [CrossRef] 
14. Sandhu, R.S.; Geojou, T.T.; Tamenbaun, A.R. Ricci curvature: An economic indicator for market fragility and system risk. Sci. Adv. 2016, 2, 1501495. [CrossRef] [PubMed]

15. Wang, Y.; Gu, X.; Chan, T.F.; Thompson, P.W.; Yau, S.T. Brain surface conformal parametrization with the Ricci flow. IEEE Trans. Med. Imaging 2012, 10, 251-264. [CrossRef] [PubMed]

16. Deshmukh, S. Almost Ricci solitons isometric to spheres. Intern. J. Geom. Methods Mod. Phys. 2019, 16, 1950073. [CrossRef]

17. Deshmukh, S.; Chen, B.Y. A note on Yamabe solitons. Balkan J. Geom. Appl. 2018, 23, 37-43.

(C) 2020 by the authors. Licensee MDPI, Basel, Switzerland. This article is an open access article distributed under the terms and conditions of the Creative Commons Attribution (CC BY) license (http://creativecommons.org/licenses/by/4.0/). 